\title{
Modified Quasi-Static, Elastic-Plastic Analysis for Blast Walls with Partially Fixed Support
}

\author{
Pattamad Panedpojaman \\ Department of Civil Engineering, Faculty of Engineering, Prince of Songkla University, Songkhla 90110, \\ Thailand \\ E-mail:ppattamad@eng.psu.ac.th
}

\begin{abstract}
Blast walls with partially fixed support have been used to limit impacts of pressure due to an explosion. As design criteria, the dynamic response of blast wall systems in terms of their pressure load-displacement relationship is considered. In addition to a complicatedly dynamic analysis, the quasi-static, elastic-plastic analysis is recently an alternative method. However, the pressure load-displacement response of the system with the short connection obtained from the quasi-static, elastic-plastic analysis tends to be over conservative comparing with the response of the previous experiments. Therefore, the quasi-static, elastic-plastic model is modified to improve the prediction accuracy. The model simply consists of two flexural elements connected through an angular spring element. The beam end is supported by the vertical and horizontal spring. The horizontal spring is simulated based on a cantilever beam mechanism and an axial restraint of the connection member. The uncoupled displacement mode shapes are assumed to represent the overall elastic-plastic behavior of the systems. The quasi-static equilibrium equations are determined from the minimum potential energy of the system. The predicted response of the modified analysis is found to better agree with the previous experimental response of the systems with the short connection.
\end{abstract}

Keywords: Blast walls, partially fixed support, quasi-static elastic-plastic analysis, pressure load-displacement response.

ENGINEERING JOURNAL Volume 16 Issue 5

Received 23 March 2012

Accepted 20 May 2012

Published 1 October 2012

Online at http://www.engj.org/

DOI:10.4186/ej.2012.16.5.45 


\section{Introduction}

Blast wall systems as shown in Fig. 1 have been used to limit impacts of pressure due to an explosion of hydrocarbon gas on temporary refuges, operation rooms of offshore oil and gas platforms and chemical laboratories. The function of blast walls is to absorb explosive energy. As a result, an efficient energy absorbing systems must be design for blast walls. Generally, a non-symmetrical trapezoidal panel is used due to its ability to absorb the energy. Guidance issued by the Fire and Blast Information Group (FABIG) and the Steel Construction Institute (SCI), known as Technical Note 5 [1], is developed to design blast walls. Note that Technical Note 5 [1] has its basis in Eurocode 3 [2]. The blast wall designs, such as moment resistance, shear resistance, local loading effect, buckling resistance, weld detail and structural dynamic response, are considered. The response of blast walls is computed in terms of their pressure loaddisplacement relationship. The pressure load is designed up to the displacement limitation.

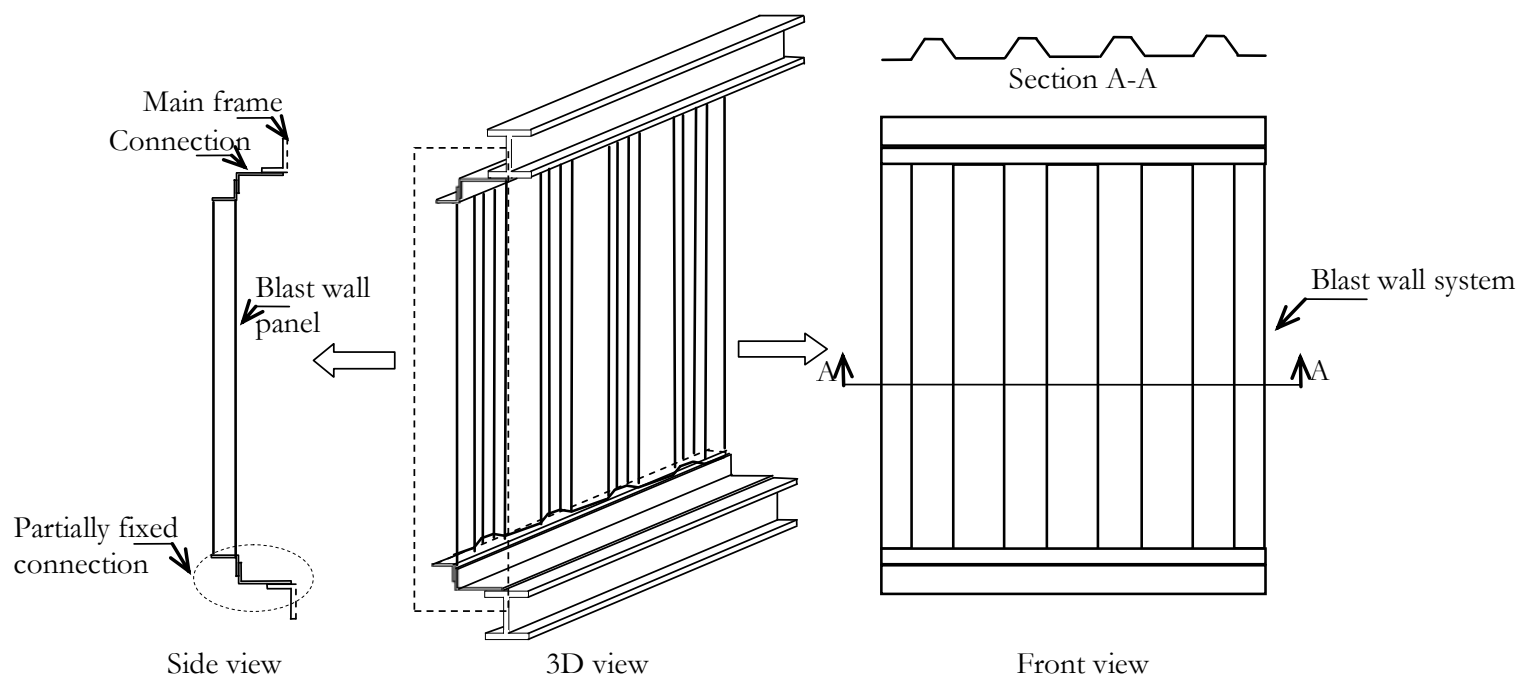

Fig. 1. Detail of blast wall systems.

The support or connection of blast walls as shown in Fig. 1 are normally considered as partially fixed behavior. However, to consider the pressure load-displacement relationship, the current designs assume a single plastic hinge formation at the ends of blast walls which is based on a simple bending model. The restrain effect of the support is ignored in the design. Therefore, the response of the wall generally tends to be conservative. Langdon and Schleyer [3] found that their experimental results of blast wall systems with the short connection provided their pressure load capacity above the standard design capacity (maintaining the displacement limitation). The results remind the actual capacity of the wall with the support is larger than that of the simple design.

Many researchers have examined methods for assessment of the response of blast walls under blast load. The finite element analysis is widely accepted for its accuracy to predict the response of blast walls [46]. However, the model is required an expensive finite element program as well as an expertise user. Approximate methods have been proposed to be an alternative design method.

Biggs [7] developed the simple design chart to approximate the structural response. The chart to predict the response is formulated based on the equivalent single-degree-of-freedom (SDOF) systems, also known as the spring-mass system. The method is well known and still used. Kaliszky [8] proposed an impulsively loaded structure consisted of an ideally rigid-plastic material and had a kinematically admissible velocity field. The impulsively loaded structure is used to be substituted with an equivalent SDOF system and be solved statically. The analysis with rigid-plastic material provides a good agreement with the elasticplastic solution when the ratio of initial kinetic energy to elastic strain energy is larger than 10, and the load duration is sufficiently short with respect to the natural period of the structure [9].

Schubak et al. [10] proposed a new procedure, a beam model, for predicting the dynamic response in consideration of axial restrain behavior and flexural behavior. The beam model is subjected to pulse loads. The elastic response of blast walls is not considered in the model. The beam model consists of two flexural elements connected through an angular spring element having rigid-plastic characteristics. To investigate 
elastic response of beams, the beam model was developed by using energy methods to provide equilibrium equations and incorporating the elastic-plastic response of the model with different structural geometries of blast wall systems [11]. Later, variable support restraints of blast walls were included in the beam model [12]. The model analysis [12] considers membrane action, strain hardening effects and flexible boundary conditions.

Due to an effect of the partially fixed connection on the overall response [3], the beam model with partially fixed connection was developed and dynamically analyzed [13]. The beam model consists of beam elements and spring elements connected to form a continuous system, as shown in Fig. 2. The spring constants used in the analysis must be pre-determined based on previous test data. The pre-determined spring constants cause limitation of using the beam model with the partially fixed connection.

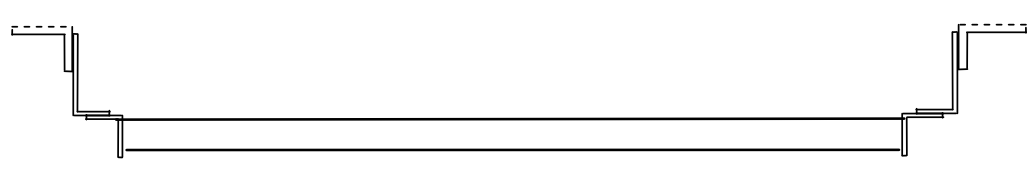

(a)

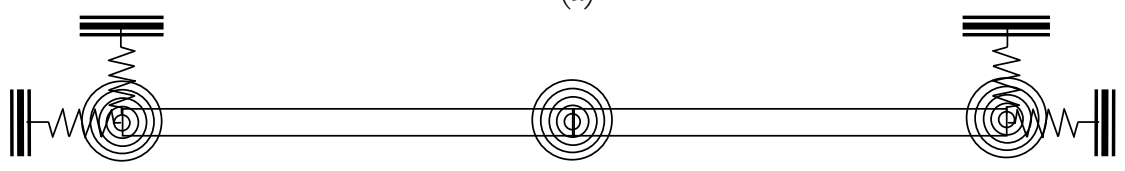

(b)

Fig. 2. Blast wall with partially fixed connection: (a) blast wall system and (b) beam model.

In addition to the complicatedly dynamic analysis, the quasi-static, elastic-plastic model [14] was recently proposed to avoid the pre-determined spring constants. The analysis is simplified by using static analysis and considered the elastic response of blast walls. Characteristics of the springs are determined based on the displacements of the beam and geometry of the connection. However, comparing with the experimental response, the predicted response of the system with the short connection is significantly conservative [14].

Therefore, this study develops the modified quasi-static, elastic-plastic analysis to improve its accuracy to predict the response of blast walls with the partially fixed support. Normally, the response is considered at the mid span which provides the maximum displacement $[3,14,15]$. The pressure-displacement at the mid span obtained from the proposed model and the previous models are investigated. The proposed model can simply be used as a part of blast wall design.

\section{Modified Quasi-Static, Elastic-Plastic Model}

The half-span blast wall system with the partially fixed support as shown in Fig. 3(a) is investigated. By using the original quasi-static, elastic-plastic model [14], the connection members are fundamentally simulated as a vertical pinned member on the both sides. However, in the modified quasi-static, elasticplastic model, the connection members fixed at the support are fundamentally simulated as a restraint angular spring based on a cantilever beam behavior. The other end is modeled as a pin at the beam end described. The fundamental model of the system described in Fig 3(b). Note that, for the full-span blast wall system, the model consists of consists of two flexural elements connected through an angular spring element at the mid-span. Subsequently, the connection member is simply modeled by the vertical and horizontal spring described in Fig 3(c). $k_{x}$ and $k_{y}$ are the horizontal and vertical spring constant, respectively. The modified quasi-static, elastic-plastic analysis to investigate the structural response is developed based on the original quasi-static, elastic-plastic equations [14]. 


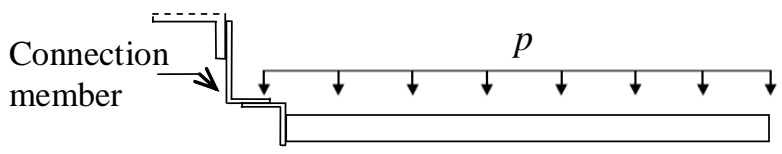

(a)

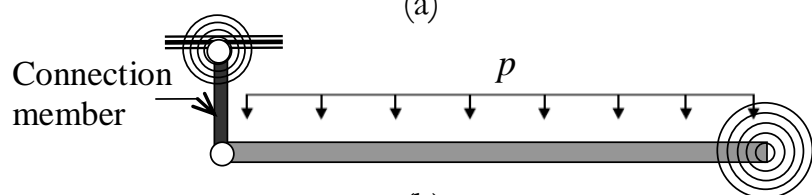

(b)

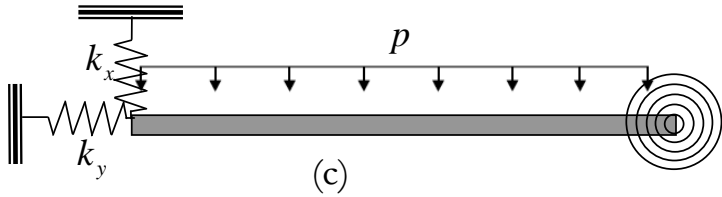

Fig. 3. Structural model of the modified quasi-static, elastic-plastic analysis: (a) blast wall system; (b) fundamental model; and (c) simplified model.

Specified to predict the overall elastic-plastic behavior, the displacement profile of the beam model under pressure loading $p$ consists of the elastic displacement mode of the connection $w_{1}(x)$, the elastic displacement mode of the beam $w_{2}(x)$ and the plastic large displacement mode $w_{3}(x)$.

$$
\begin{aligned}
& w_{1}(x)=C_{1} \\
& w_{2}(x)=C_{2} \cos \pi x / 2 L \\
& w_{3}(x)=C_{3}(1-x / L)
\end{aligned}
$$

in which $C_{1}, C_{2}$ and $C_{3}$ are the maximum displacement of each mode as shown in Fig. 4; and $L$ is the halfspan length of the system. The uncoupled mode shapes allow to separately determining the elastic and plastic effects in the quasi-static analysis. The mode shapes are used to formulate the total energy due to flexural and membrane behavior of the structural elements. The structural response is analyzed based on a consideration of the minimum potential energy of the beam system. As a result, a complex mechanism can be simplified in the analysis.

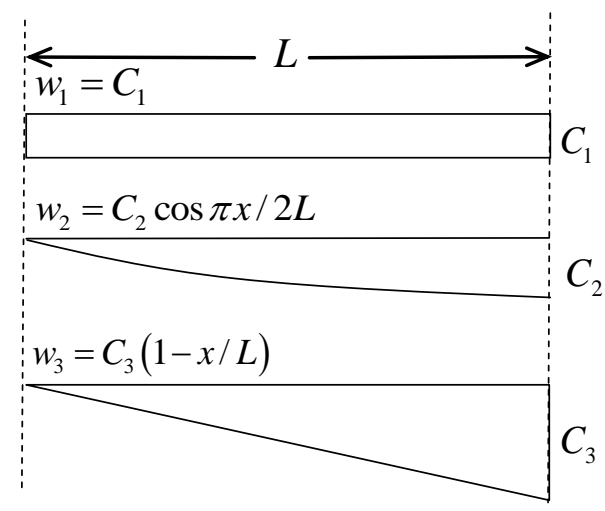

Fig. 4. Displacement mode shapes of the beam model

\subsection{Elastic Stage}

Under the elastic stage of the beam, the displacement profile combines the fundamental flexural mode shape of a simply supported beam and the vertical support displacement. The axially restraint (i.e., 
horizontal restraint) at the beam end is assumed to be neglected due to its small displacement. The shape function $w(x)$ is approximated as

$$
w(x)=C_{1}+C_{2} \cos \pi x / 2 L
$$

For the half-span blast wall system, the flexural strain energy of the beam element $U_{b}$, the potential energy loss $P E$ due to the loading $p$ and the total potential energy $V$ of the system are given as follows:

$$
\begin{aligned}
& U_{b}=\int_{0}^{L} \frac{M(x)^{2}}{2 E I} d x=\frac{E I}{2} \int_{0}^{L}\left(\frac{d^{2} w}{d x^{2}}\right)^{2} d x \\
& P E=-\int_{0}^{L} p w(x) d x \\
& V=\sum U+P E=U_{b}+P E
\end{aligned}
$$

in which $M(x)$ is the bending moment; $E$ is the Young's modulus of elastic; and $I$ is the second moment of area of the blast wall panel. The quasi-static equilibrium equations are determined from the minimum potential energy of the system which is

$$
\frac{\partial V}{\partial C_{i}}=0
$$

Eq. (8) provides the formulation of $C_{1}$ and $C_{2}$ as follows:

where

$$
\begin{aligned}
C_{1} & =\frac{p L}{k_{y}} \\
C_{2} & =\frac{64 p L^{4}}{E I \pi^{5}} \\
k_{y} & =\frac{A_{c n t} E}{L_{c n t}}
\end{aligned}
$$

$A_{c n t}$ and $L_{c n t}$ are the area and the length of the connection member.

Limited by the plastic moment capacity $M_{p}$ of the beam at the mid-span, the maximum elastic displacement of the beam $\mathrm{C}_{2}{ }^{\max }$ is given in Eq. (12). The corresponding load at the elastic limiting $p_{1}$ can be computed in Eq. (13).

$$
\begin{gathered}
C_{2}^{\max }=\frac{4 M_{p} L^{2}}{E I \pi^{2}} \\
p_{1}=\frac{E I \pi^{5} C_{2}^{\max }}{64 L^{4}}
\end{gathered}
$$

\subsection{Plastic Stage}

The plastic deformation of the beam after plastic hinge formation at the mid-span is approximated by the shape function $w_{3}(x)$. Due to the large displacement of the plastic hinge, the connection member is assumed to stretch and rotate as shown in Fig. 5(a). The beam ends are considered to be restrained both axially and vertically due to the resistance of the connection in terms of the horizontal force $F_{x}$ and the vertical force $F_{y}$. Movement of the corresponding connection spring is described in Fig. 5(b). The movement is assumed to less affect the overall large displacement of the beam. 


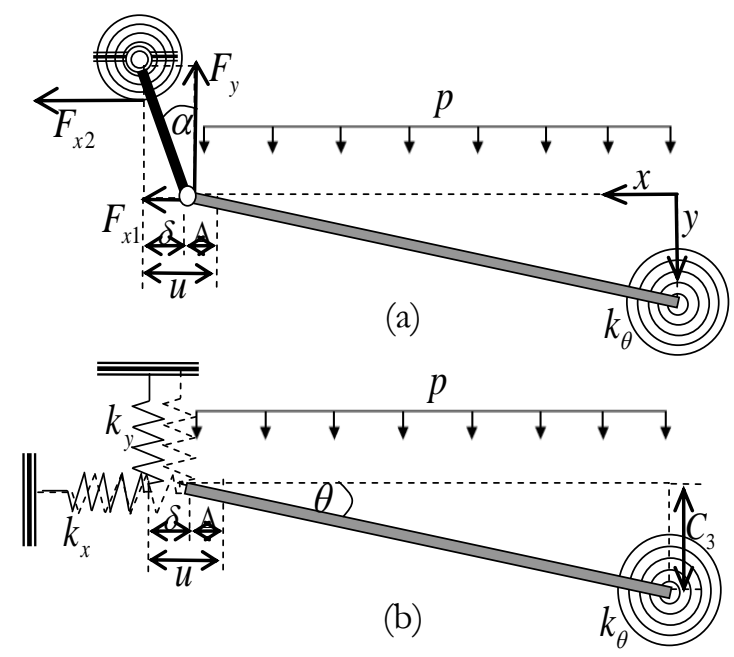

Fig. 5. Large displacement geometry and forces of connection: (a) fundamental model, and (b) simplified model.

From the large displacement geometry of the blast wall systems in Fig. 5(b), the horizontal displacement $u$ corresponding to $w_{3}(x)$, the axial stretching of the beam during bending $\Delta$, the horizontal movement of the connection $\delta$ and the angle of the connection rotation $\alpha$ are formulated as follows:

$$
\begin{aligned}
& u=L-\sqrt{(L)^{2}-\left(C_{3}\right)^{2}} \\
& \Delta=\frac{F_{x} L}{A E} \\
& \delta=u-\Delta \\
& \alpha=\sin ^{-1}\left(\frac{\delta}{L_{c n t}}\right) \approx \sin ^{-1}\left(\frac{u}{L_{c n t}}\right)
\end{aligned}
$$

where $A$ is the area of the blast wall panel. Since the value of $\Delta$ is normally very small comparing with the value of $u$, the value of $\delta$ can be approximated to be same as the value of $u$ in Eq. (17).

Based on the vertical force equilibrium, the resistance force of the connection in $y$ direction $F_{y}$ is evaluated as

$$
F_{y}=p L
$$

whereas $F_{x}$ is depended on the combination of the axial restraint $F_{x 1}$ in Eq. (19) and the restraint of the angular spring $F_{x 2}$ based on the cantilever beam mechanism in Eq. (20). Due to blast wall systems are generally designed to failure at its blast wall panel such as in the buckling mode [3], $F_{x 2}$ is assumed to be only in the elastic stage. Therefore, $F_{x}$ and the corresponding $k_{x}$ is given in Eq. (21) and Eq. (22), respectively.

$$
\begin{aligned}
& F_{x 1}=F_{y} \tan \alpha=p L \tan \alpha \\
& F_{x 2}=\frac{3 E I \delta}{L_{c n t}^{3}} \\
& F_{x}=F_{x 1}+F_{x 2}
\end{aligned}
$$




$$
k_{x}=\frac{F_{x}}{\delta}
$$

$F_{x}$ in Eq. (21) is evaluated based on compatibility of Eq. (15) and Eq. (16). A trial and error process is required to solve $F_{x}$.

To consider the total potential energy, the strain energy of the beam element $U_{m, b}$ and the horizontal spring element $U_{m, s x}$ are computed in Eq. (23) and Eq. (24). The angular spring element can be assumed as a rigid-plastic characteristic in the model [14], as shown in Fig. 6. The strain energy of the angular spring at the mid-span $U_{\theta}$ is computed in Eq. (25).

$$
\begin{aligned}
& U_{m, b}=\frac{\Delta^{2} A E}{2 L} \\
& U_{m, s x}=\frac{\delta^{2} k_{x}}{2} \\
& U_{\theta}=M_{p} \theta+\frac{k_{\theta} \theta^{2}}{2}
\end{aligned}
$$

in which $k_{\theta}$ is the angular spring stiffness of hardening moment due to hardening strain; and $\theta$ is the angle of the beam rotation, as described in Fig. 5, and can be numerically approximated as $C_{3} / L$. The total potential energy $V$ of the system at the plastic stage is

$$
V=\sum U+P E=U_{m, b}+U_{m, s x}+U_{\theta}+P E
$$

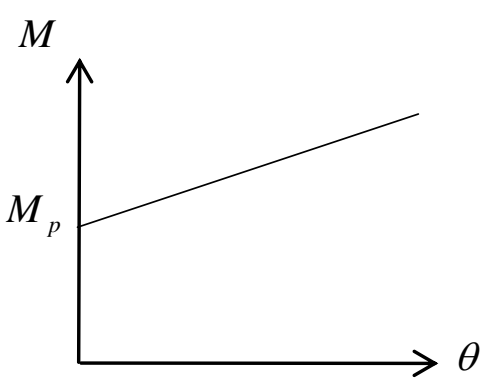

Fig. 6. Rigid-plastic characteristics.

The minimum potential energy of $\partial V / \partial C_{3}=0$ provides the relationship between $p$ and $C_{3}$ as

$$
p=\beta_{1} \frac{C_{3}^{3}}{L^{4}}+\beta_{2} \frac{C_{3}^{3}}{L^{3}}+\frac{2 M_{p}}{L^{2}}+\frac{2 k_{\theta} C_{3}}{L^{3}}
$$

where

$$
\begin{aligned}
& \beta_{1}=\frac{A E}{\left[1+\frac{A E}{k_{x} L}\right]^{2}} \\
& \beta_{2}=\frac{k_{x}}{\left[1+\frac{k_{x} L}{A E}\right]^{2}}
\end{aligned}
$$


Note that $\beta_{1}$ and $\beta_{2}$ are numerically solved by using Binomial series of the first two terms. The relationship between $p$ and $C_{3}$ is solved based on a trial and error process. In the plastic stage, the overall displacement at the mid span can be considered as $w(x=0)=C_{1}+C_{2} \max +C_{3}$. Following the procedure in the elastic and plastic stage, the relationship between $p$ and $w(x)$ can also be completely plotted.

\section{Quasi-Static Loading Regime}

To adopt the quasi-static analysis, the structural response must be classified in the quasi-static loading regime. Biggs [7] investigated the structural response of blast wall systems under a pulse load having a triangular shaped pressure pulse with a duration $t_{d}$. The single-degree-of-freedom (SDOF), elastic springmass system is used in the investigation. The study describes that dynamic effects significantly influence on the response of blast wall systems when $t_{d} / T<1$ in which $T$ is the fundamental elastic period of vibration of blast wall systems. At the same peak load, the static displacement calculated agrees with the dynamic displacement within approximately $17 \%$ when $t_{d} / T>1.75$. Inertia effects are significant when the loading is applied almost instantaneously as in the case of shock loading.

The structural response of blast walls under a blast load is influenced by the ratio $t_{d} / T$ or $\omega t_{d}$ (i.e. $\omega t_{d}=$ $2 \pi t_{d} / T$ ). Three loading regimes are classified as follows [14]: quasi-static loading regime for $\omega t_{d}>40$, dynamic loading regime for $0.4<\omega t_{d} \leq 40$, and impulsive loading regime for $\omega t_{d} \leq 0.4$. The fundamental elastic period $T$ of vibration of a blast wall is given as

where

$$
\begin{aligned}
T & =2 \pi \sqrt{M / K} \\
M & =k_{M} m \\
K & =k_{L} k \\
k & =\frac{384 E I}{5(2 L)^{3}}
\end{aligned}
$$

$M$ and $K$ are the equivalent mass and the equivalent stiffness of blast wall systems for the SDOF analysis, respectively. $m$ and $k$ are the mass and the elastic spring constant of blast wall systems, respectively. The formulation of $k$ in Eq. (33) is evaluated based on blast wall systems considered as a simple support with no end fixity and a uniformly distributed loading. $k_{M}$ is the mass transformation factor which is 0.50 for the elastic stage and 0.33 for the plastic stage [7]. $k_{L}$ is the load transformation factor which is 0.64 for the elastic stage and 0.50 for the plastic stage [7]. Through the above equations, the loading regime can be classified.

\section{Validation of the Modified Analysis}

To validate the proposed model, the blast wall system with the connection lengths $L_{c n}$ is investigated as shown in Fig. 7. The connection lengths $L_{v n t}$ of $240 \mathrm{~mm}, 480 \mathrm{~mm}$ and $720 \mathrm{~mm}$ are specified in the analysis and denoted as the short, medium and long connection through the criteria of $L_{c u t} / L \leq 0.12,0.12<L_{c n t} / L$ $<0.36$ and $L_{c n t} / L \geq 0.36$, respectively [13]. The structural response of the system is described in terms of the pressure load-displacement curve at the mid span. The curves are compared with a series of the experimental investigation [3, 15], the finite element analysis [14] and the original quasi-static, elastic-plastic analysis. The blast wall panel properties are illustrated in Table 1. The blast wall is made of 316L stainless steel.

Note that the study approximates the hardening moment of resistance in Table 1 through the pressuredisplacement curve of the FE analysis [14]. The value of the hardening moment of resistance can also be evaluated from experiments. However, the previous experiments are conducted by using the $1 / 4$ scale blast walls. Therefore, the value in this study is approximated through the pressure-displacement curve of FE analysis. Without the hardening moment of resistance, the pressure-displacement curve based on Eq. (29) is over conservative.

The previous investigations were conducted through the blast wall systems under pulse pressure load in $[3,14]$ as shown in Fig. 8 and under static pressure load in [15]. Due to limitation of the full scale test, the experimental investigations $[3,15]$ were conducted through the $1 / 4$ scale blast walls. For comparing the 
response of the full scale blast wall with that of the $1 / 4$ scale blast wall [14], the displacement of the $1 / 4$ scale blast wall is scaled up which is multiplied by 4 whereas the pressure values are not changed. The detail of scaling up the $1 / 4$ scale blast walls is described in [14]. All pulse pressure loads in [3,14] are considered as the quasi static load based on the classification of the structural response.

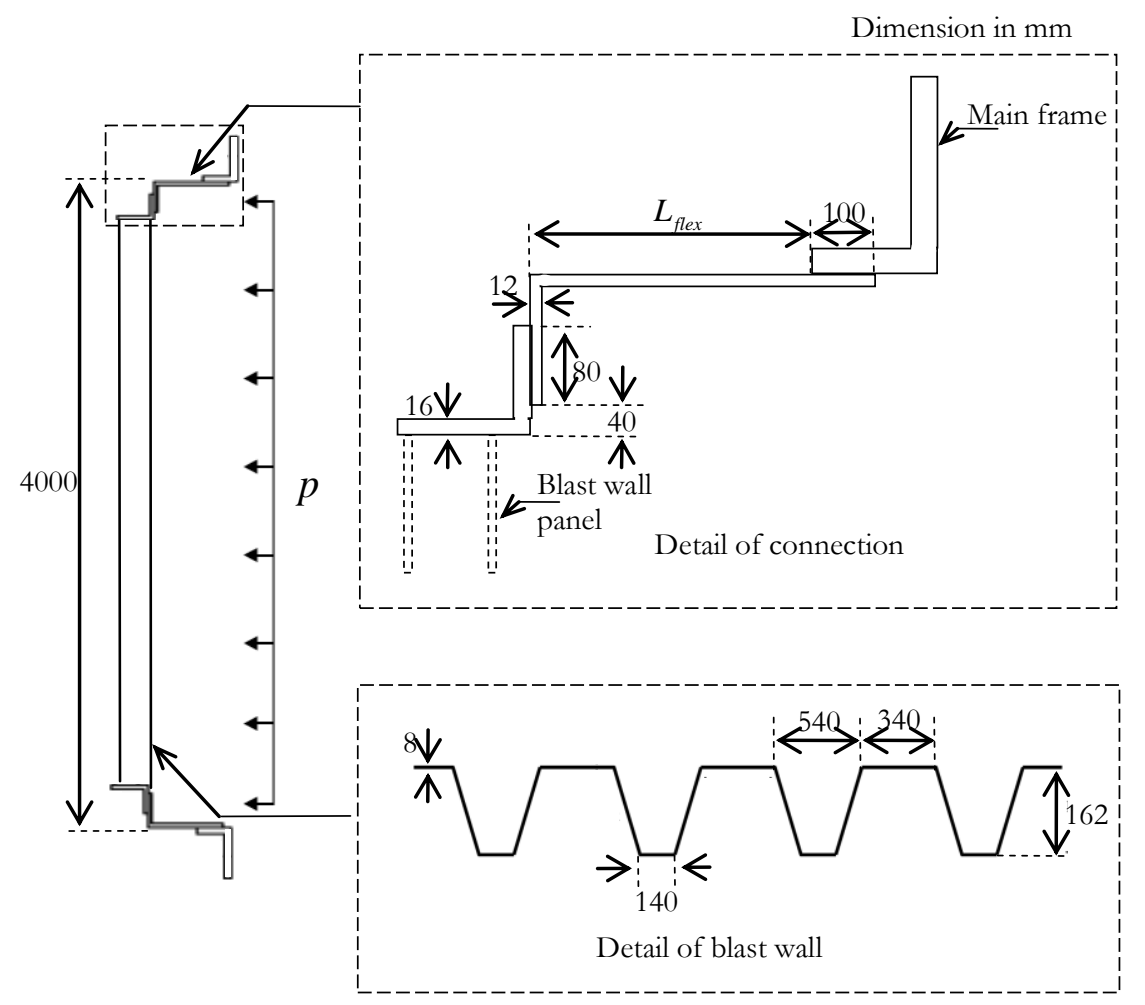

Fig. 7. Detail of the investigated blast wall system.

Table 1. Blast wall panel properties [14].

\begin{tabular}{lll}
\hline Properties & \multicolumn{2}{l}{ Magnitude } \\
\hline Cross-section area of profile & 8598.0 & $\mathrm{~mm}$ \\
Profile material density & 7970.0 & $\mathrm{~kg} / \mathrm{m}^{3}$ \\
Mass of beam section/unit length & 68.5 & $\mathrm{~kg} / \mathrm{m}$ \\
Second moment of area & 3638.0 & $\mathrm{~cm}$ \\
Young's modulus & 200.0 & $\mathrm{~N} / \mathrm{mm}^{2}$ \\
Initial plastic moment & 151.5 & $\mathrm{kNm}$ \\
Hardening moment of resistance & 455.0 & $\mathrm{kNm} / \mathrm{rad}$ \\
Base yield stress & 265.0 & $\mathrm{~N} / \mathrm{mm}^{2}$ \\
Design dynamic yield stress & 311.4 & $\mathrm{~N} / \mathrm{mm}^{2}$ \\
\hline
\end{tabular}

By using the quasi-static, elastic-plastic analysis, the analyzed pressure load corresponding to the initial plastic moment of the blast wall is 0.75 bar. Such pressure load is found to differ from the experiments which are about 0.91 bar. The reason may due to the design dynamic yield stress of $311.4 \mathrm{~N} / \mathrm{mm}^{2}$ does not represent the real value of the experiment. The dynamic yield stress normally depends on details of its dynamic test. Based on the experiment of [16], the dynamic yield stress of $316 \mathrm{~L}$ stainless steel varies from $300 \mathrm{~N} / \mathrm{mm}^{2}$ to $400 \mathrm{~N} / \mathrm{mm}^{2}$. Therefore, to lessen the effect of the various dynamic yield stresses, the normalized pressure load $p / p_{1}$ is used in the comparison. 


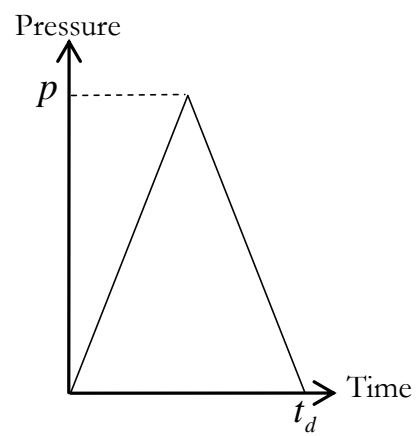

Fig. 8. Triangular pulse load.

Comparisons of the normalized pressure-displacement curve at the mid span between the proposed model and the original model are shown in Fig. 9. For the blast wall system with the medium or long connection, it is found that the curves obtained from the modified analysis slightly differ from the original method. In other words, the horizontal resistance due to the cantilever beam model less affects the pressure resistance of the system with the medium or long connection. Furthermore, the modified analysis and the original method agree well with the previous experiments both static and impulse load. However, for the blast wall system with the short connection, the cantilever beam model of the connection is significantly improved the pressure resistance of the systems. Comparing with the previous investigations, the modified analysis provides more accuracy to predict the structural response of the blast wall systems.
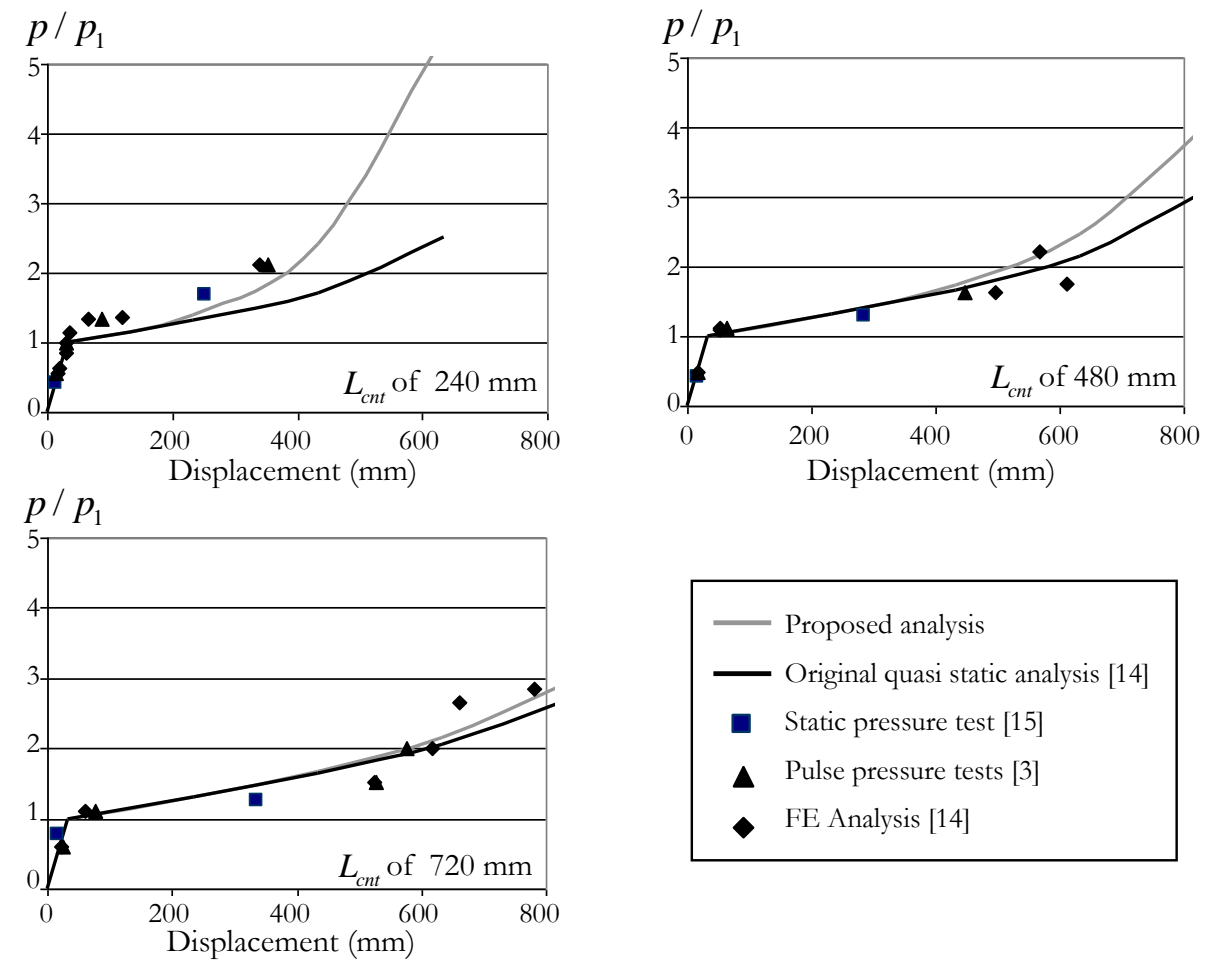

Fig. 9. Comparisons of the normalized pressure -displacement curve.

\section{Conclusion}

To predict the pressure load-displacement response of blast wall systems, the study proposes the modified quasi-static, elastic-plastic analysis. The fundamental model of the quasi-static, elastic-plastic analysis consists of two flexural elements connected through an angular spring element. The angular spring element at the mid-span is simulated based on the rigid-plastic behavior. The beam support is simulated as the vertical and horizontal spring. The horizontal spring resistance is formulated through a cantilever beam 
mechanism and an axial restraint of the connection member. The displacement mode shapes of the elastic displacement mode of the connection, the elastic displacement mode of the beam and the plastic large displacement mode are used to analyze the overall elastic-plastic behavior. The uncoupled mode shapes allow to separately determining the elastic and plastic effects in the quasi-static analysis. The total energy of flexural and membrane behavior is derived by using the displacement of the model corresponding to the mode shapes. The structural response is analyzed based on a consideration of the minimum potential energy of the beam system. The pressure load-displacement response is solved through a trial and error process.

To validate the modified analysis, the normalized pressure-displacement curve at the mid span is used to lessen the effect of the various dynamic yield stresses. The predicted pressure resistance at a given displacement of the blast wall system with the short connection is found to be significantly improved comparing with that of the original model. The predicted response also agrees with the experimental results. However, the modified analysis less affects the response of the systems with the medium or long connection.

The comparison describes the modified analysis is required to better predict the response of the blast wall systems with the short connection. The modified quasi-static, elastic-plastic analysis can be considered as a fundamental tool of blast wall design.

\section{Acknowledgement}

We would like to sincerely thank all the journal reviewers who dedicated their time to provide valuable comments.

\section{References}

[1] Fire and Blast Information Group (FABIG), "Design guide for stainless steel blast walls," Technical Note 5, SCI, June, 1999.

[2] CEN, "Design of steel structures, Part 1-3, supplementary rules for cold-formed thin gauge members and sheeting," ENV 1993-1-3, 1996.

[3] G. S. Langdon, and G. K. Schleyer, "Inelastic deformation and failure of profiled stainless steel blast walls. Part I: experimental investigations," Int. J. Impact Eng., vol. 31, pp. 341-369, 2005.

[4] L. A. Louca, J. W. Boh, and Y. S. Choo, "Design and analysis of stainless steel profiled blast barriers," J. Constr. Steel Res., vol. 60, pp. 1699-1723, 2004.

[5] W. Jun, L. Jingbo, Y. Qiushi, "Effect of shock wave on fabricated anti-blast wall and distribution law around the wall under near surface explosion," Trans. Tianjin Univ., vol. 14 pp. 514-518, 2008.

[6] M. A. Faruqi, J. Grisel, A. Salem, and J. Sai, "A parametric study for the efficient design of corrugated blast wall panels used in petrochemical facilities," ARPN J. Eng. Appl. Sci., vol. 5, no. 11, pp. 89-96, 2010.

[7] J. M. Biggs, Introduction to Structural Dynamics, NewYork, McGraw-Hill, 1964.

[8] S. Kaliszky, "Large deformations of rigid-plastic structures under impulsive and pressure loading," $J$. Struct. Mech., vol. 1, no. 3, pp. 295-317, 1973.

[9] N. Jones, Structural Impact, Cambridge, Cambridge University Press, 1989.

[10] R. B. Schubak, D. L. Anderson, and M. D. Olson. "Simplified dynamic analysis of rigid-plastic beams," Int. J. Impact Eng., vol. 8, no.1, pp. 27-42, 1989.

[11] G. K. Schleyer, and M. Mihsein, "Development of mathematical models for dynamic analysis of structures," Conference Proceedings on Structural Design against Accidental Loading-As Part of the Offshore Safety Case, London, pp. 3.2.1-11, 1992.

[12] G. K. Schleyer, and S. S. Hsu, "A modelling scheme for predicting the response of elastic-plastic structures to pulse pressure loading," Int. J. Impact Eng., vol. 24, pp. 759-77, 2000.

[13] G. S. Langdon, and G. K. Schleyer, "Inelastic deformation and failure of $1 / 4$ scale profiled stainless steel blast wall panels. Part II: analytical modelling considerations," Int. J. Impact Eng., vol. 31, no. 4, pp. 371-399, 2005.

[14] G. K. Schleyer, and G. S. Langdon, "Pulse pressure testing of $1 / 4$ scale blast wall panels with connections Phase II," HSE Research Report 404, 2006. 
[15] G. S. Langdon, and G. K. Schleyer, "Response of Quasi-statically Loaded Corrugated Panels with Partially Restrained Boundaries," Exper. Mech., vol. 47, pp. 251-261, 2007.

[16] G. S. Langdon, and G. K. Schleyer, "Unusual strain rate sensitive behaviour of AISI 316L austenitic stainless steel," IMechE J. of Strain Analysis, vol. 39, no. 1, pp. 71-86, 2004. 